\title{
Spectral characterization of SPDC-based single-photon sources for quantum key distribution
}

\author{
Sabine Euler, Erik Fitzke, Oleg Nikiforov, Daniel Hofmann, Till Dolejsky, and Thomas Walther ${ }^{\mathrm{a}}$ \\ Institute for Applied Physics, TU Darmstadt, Schlossgartenstr. 7, 64289 Darmstadt, Germany
}

Received 18 May 2020 / Accepted 8 January 2021 / Published online 26 April 2021

(C) The Author(s) 2021

\begin{abstract}
In our laboratory, we employ two biphoton sources for quantum key distribution. The first is based on cw parametric down-conversion of photons at $404 \mathrm{~nm}$ in PPKTP waveguide chips, while the second is based on the pulsed parametric down-conversion of $775 \mathrm{~nm}$ photons in PPLN waveguides. The spectral characterization is important for the determination of certain side-channel attacks. A Hong-Ou-Mandel experiment employing the first photon source revealed a complex structure of the common Hong-OuMandel dip. By measuring the spectra of the single photons at $808 \mathrm{~nm}$, we were able to associate these structures to the superposition of different transverse modes of the pump photons in our waveguide chips. The pulsed source was characterized by means of single-photon spectra measured by a sensitive spectrum analyzer as well as dispersion-based measurements. Finally, we also describe Hong-Ou-Mandel experiments using the photons from the second source.
\end{abstract}

\section{Introduction}

Cryptography is at the heart of today's technology. Basically all transfer of data over the internet is encrypted via the RSA algorithm: Two parties employ a public and a private key to keep any information safe. While encrypting the message using the public key is possible for everyone, only the recipient of the message holding the private key is able to decrypt the message properly. Decrypting the message without the private key, i.e. computing the private key from the public key, requires the prime factorization of large numbers. As this is a complex problem for any classical computer, the RSA algorithm is complexity-theoretic secure. However, the security of public key infrastructure is potentially threatened by the advent of the quantum computer. It can be proven that the Vernam one-time-pad, which is a random key of the same length as the message and only used once, provides information-theoretically secure communication. Thus, the problem remaining is the distribution of this key.

A possible solution to this challenge is quantum key distribution (QKD). It has gained a lot of interest in recent years as it can provide such a method to distribute a secret key between two parties referred to as Alice and Bob. Moreover, due to the no-cloning theorem, it is possible to identify whether an eavesdropper ("Eve") has tried to acquire the key or parts of it. This is an entirely new property compared to classical sym-

\footnotetext{
${ }^{a}$ e-mail: thomas.walther@physik.tu-darmstadt.de (corresponding author)
}

metric key distribution. Moreover, QKD allows for a long term secure communication storage of data [1].

Today, a variety of QKD protocols exist and first companies already offer QKD systems on the market $[2,3]$. Usually, photons are used as the quantum information carriers. They can be easily distributed over optical fibers or free-space optical links.

QKD protocols use the different degrees of freedom of photons to encode the quantum information, such as polarization, phase and time. Some QKD protocols use entangled photon pairs, generated e.g. by spontaneous parametric down-conversion (SPDC) $[4,5]$ or spontaneous four-wave mixing $[6,7]$. As commercial applications demand for high key rates, wavelength multiplexing may also be used to increase the key rates, simultaneously making the wavelength spectrum of the photons a subject of interest.

While QKD provides unconditional security in an ideal implementation, practical implementations might suffer from shortcomings rendering them exploitable against certain attacks [8]. Some of these attacks might be side-channel attacks, i.e., attacks that are not directly linked to the quantum channel. Examples for such side channels are, among others, power consumption or the wavelength of the photons used in the communication.

In this paper, we discuss the investigation of two of our photon sources for QKD experiments based on spontaneous parametric down-conversion (SPDC) in waveguide chips manufactured by AdvR, Inc. in a typeII down-conversion process. The first source is pumped by a single-mode laser diode at $404 \mathrm{~nm}$ and operates at around $808 \mathrm{~nm}$ for free-space QKD based on the 
Fig. 1 Single photon spectra generated by SPDC in the NIR. The dashed vertical line in the spectra corresponds to double the wavelength of the pump radiation. A rich structure is revealed strongly depending on the selected pump mode depicted in the image above the spectra. The waveguide chip has a multimode structure for $404 \mathrm{~nm}$, but is single mode for the NIR photons
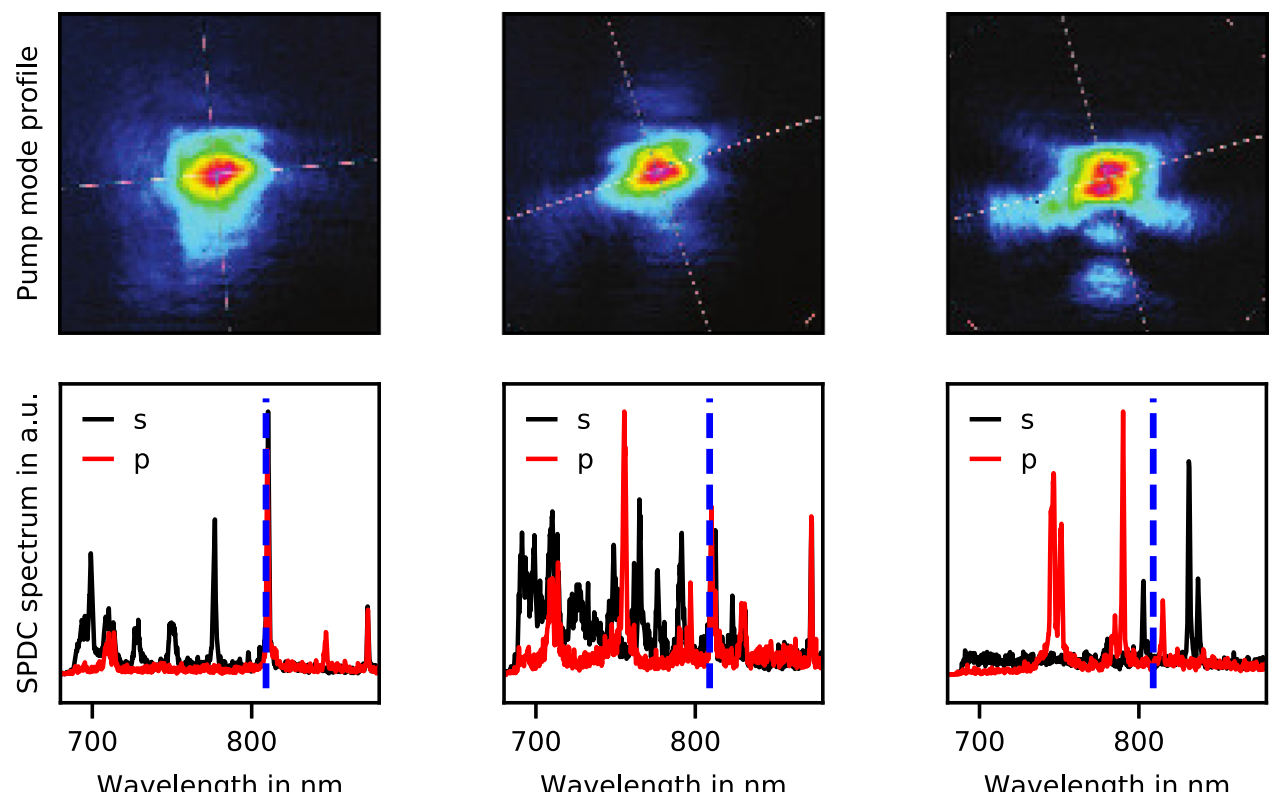

Wavelength in $\mathrm{nm}$

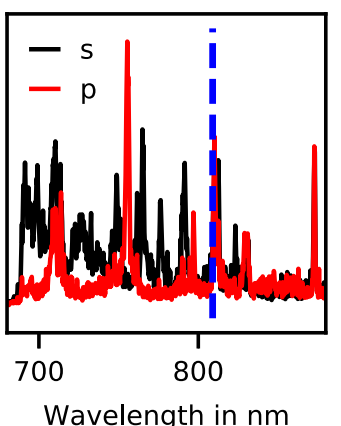

BB84 protocol. The PPKTP waveguides have a length of $4 \mathrm{~mm}$ and $11 \mathrm{~mm}$ and are between $3 \mu \mathrm{m}$ and $8 \mu \mathrm{m}$ wide. They are single-mode for the NIR, but multimode for the $404 \mathrm{~nm}$ pump.

The second is a fiber-based source for QKD compatible with telecom wavelengths around $1550 \mathrm{~nm}$. The waveguide chips are $24 \mathrm{~mm}$ long PPLN chips with $10 \mu \mathrm{m}$ wide waveguides. While ultimately it is geared towards a WDM based ansatz for a quantum hub, understanding the sources is the key. Therefore, we address in this paper the spectral characterization of the photons generated in our sources.

We investigate the spectra of the down-converted photons by spectrographs capable of single-photon detection in both cases. Depending on the pump mode, i.e., alignment of the $404 \mathrm{~nm}$ pump, the spectra look decisively different, as shown in Fig. 1. The other studies were performed with Hong-Ou-Mandel interferometers [9] and a setup with similarity to the HanburyBrown-Twiss interferometer, i.e., the Shih-Alley interferometer [10].

\section{Photon characterization around $808 \mathrm{~nm}$}

As our free space implementation of the BB84 protocol required degenerate photon pairs at $808 \mathrm{~nm}$, we set up a Hong-Ou-Mandel (HOM) interferometer to make sure that the $808 \mathrm{~nm}$ source actually produced degenerate photon pairs.

In the HOM interferometer, two photons impinge on two different inputs of a beam splitter, while in the two exits of the beam splitter coincident events are recorded, i.e., the number of photon pairs that are separated by the beam splitter. As long as both photons are distinguishable, this separation is a probabilistic event. Half of the photon pairs will be separated, the other half will jointly exit the beam splitter. The HOM interference is an effect of indistinguishable photon pairs: both photons will bunch, they will stick together with certainty, coincident events in the different outputs will decrease to zero.

In the $808 \mathrm{~nm}$ setup the two down-converted photons are generated in a type-II process, i.e., they are orthogonally polarized. Thus, they are separated by a polarizing beam splitter and guided by polarization maintaining alignment fibers to a free-space Hong-Ou-Mandel interferometer (HOMI). One path of the interferometer can be changed in length as to make the photons distinguishable by different arrival times on the beam splitter. Coincident events in the outputs of the beam splitter are detected by two avalanche photo diodes (APD). The polarization of the two photons in the interferometer is adjusted by two half-wave plates.

The well known Hong-Ou-Mandel dip is visible once the two photons are degenerate and impinge simultaneously onto the beam splitter, i.e., when they are indeed indistinguishable. It is interesting to note that the shape of the dip depends on the spectral properties of the two photons. Two possible shapes are depicted in Fig. 2b (middle) along with the corresponding experimental spectral shape (left column of Fig. 2b). While the overall shape is as expected (right column of Fig. 2b), we found subtle deviations clearly visible due to the high $S / N$ ratio in our measurements.

In order to further investigate these deviations, we set up a second two-photon interferometer according to Shih and Alley [10]. Here, the two photons of a pair impinge on the same input of a beam splitter, the separation of the photon pairs is random in every setup. However, indistinguishable photon pairs separated by the beam splitter are projected onto a polarization entangled state described below. Our type-II $808 \mathrm{~nm}$ photons are guided to the interferometer setup by a polarization maintaining fiber. A Michelson type 


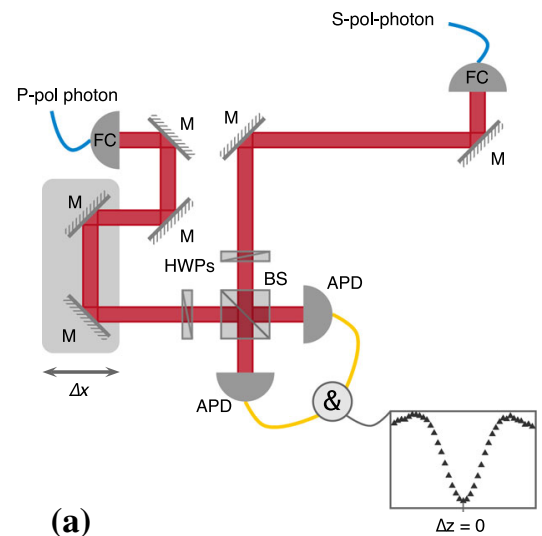

(a)

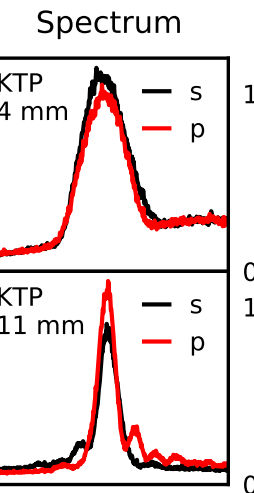

(b)
Expectation

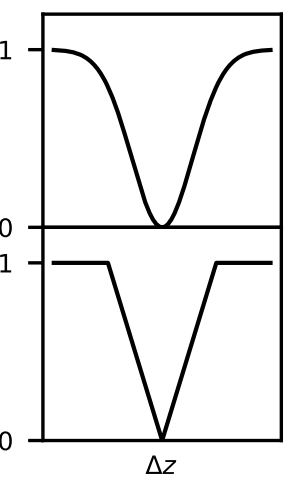

Experiment

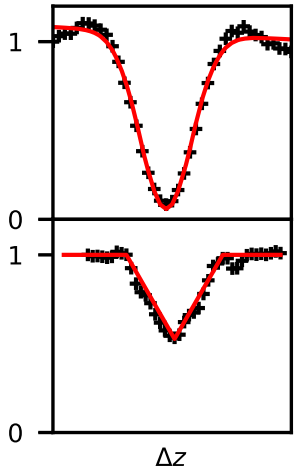

Fig. 2 a Setup of the Hong-Ou-Mandel experiment for the $808 \mathrm{~nm}$ photons. $F C$ fiber coupler, $A P D$ single-photon detectors, $H W P$ half-wave plate, $B S$ beam splitter, $M$ mirror. b Spectral shape (left) for the SPDC photons $\mathrm{w} / 3 \mathrm{~nm}$ bandpass filter and $4 \mathrm{~mm}$ waveguides, s-s-polarized, p-p- polarized (top), spectrum for the SPDC photons with $11 \mathrm{~mm}$ w/o filter (bottom), (middle) expected HOM dips, (right) actual HOM dips with subtle differences to the expectation

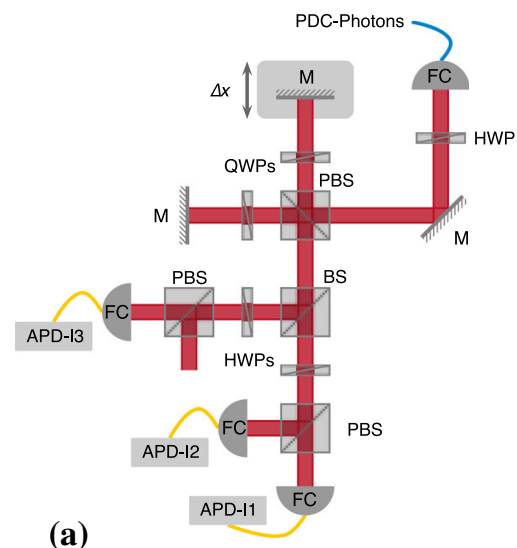

(a)
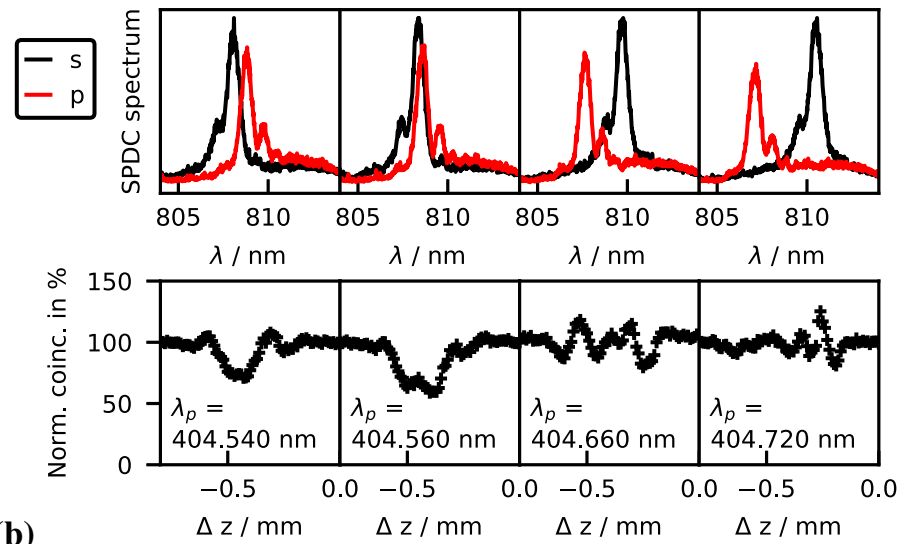

(b)

$\Delta \mathrm{z} / \mathrm{mm}$

$\Delta \mathrm{z} / \mathrm{mm}$

Fig. 3 a Setup of the Shih-Alley experiments. $F C$ fiber coupler, $A P D$ single-photon detectors, $Q W P / H W P$ quarter/halfwave plate, $P B S$ polarizing beam splitter, $B S$ beam splitter, $M$ mirror $\mathbf{b}$ results

interferometer is used to induce a time delay between the two photons of a pair. They are separated by a polarizing beam splitter cube and recombined at the same cube after the double pass of two quarter-wave plates. By shifting the path length of one photon with respect to the other one before recombination, the relative arrival time at the Shih-Alley interferometer (SAI) is controlled. Upon entering the SAI both photons impinge onto a beam splitter (cf. Fig. 3a).

To observe the Shih-Alley interference, a half-wave plate as well as a PBS are placed in each of the outputs of the beam splitter. Two APDs register coincidences between these two outputs which exhibits interference between the two photons. For a parallel orientation of the analyzers one cannot detect any coincidences, whereas a perpendicular orientation actually produces a maximum. The expected shape (Gaussian or triangular) of the curve is equivalent to that of the HOM interferometer.

Figure $3 \mathrm{~b}$ shows typical SAI spectra for different down-converted spectra. In order to control the energytime state of the signal and idler photon, the phase matching condition of the PDC process has to be manipulated. This can be done by changing the temperature of the chip or the wavelength of the pump radiation. As shown, we opted for different pump wavelengths. The top row shows the spectra of the downconverted s-polarized and p-polarized photons, the bottom row shows the results of the corresponding SAI correlation measurements. The SAI data show a rich interference structure with regions showing bunching (above 100\%) and anti-bunching (below 100\%).

Figure 4 explains this in more detail. Again, we show a spectrum on the left and the corresponding SA interferogram on the right. The spectra show the s-polarized and p-polarized down-converted photons. Clearly, there are spectral contributions which might be either spolarized or p-polarized. They originate from different pump modes; thus, each fulfilling a slightly different phase matching condition. This behavior is possible due to the multi-mode character of the waveguides for the pump radiation. The pump wavelength and the crystal temperature have been chosen such that the s-polarized idler is degenerate with the p-polarized signal from the 
other mode. Thus, we have a photon state which can be described by the entangled state

$$
|\Psi\rangle=\frac{1}{\sqrt{2}}\left(c\left|\uparrow \omega_{i}, \leftrightarrow \omega_{s}\right\rangle+\mathrm{e}^{\mathrm{i} \phi} d\left|\leftrightarrow \omega_{i}, \uparrow \omega_{s}\right\rangle\right)
$$

with angular frequencies $\omega_{i}=\omega_{0}-\frac{\Delta \omega}{2}, \omega_{s}=\omega_{0}+\frac{\Delta \omega}{2}$, polarization states $\uparrow$ and $\leftrightarrow$ and real coefficients $c, d$, $\omega_{0}$ is half the pump frequency and $\hbar \Delta \omega$ is the energy difference between signal and idler photon. This entanglement is unique to the type-II SPDC process under consideration and can explain the rich structure of our SAI results. In order to simplify the discussion, we only consider a state with a spectrum consisting essentially of only two idler/signal contributions. After separation at the polarizing beam splitter and propagation in the SA interferometer, the input state at the final beam splitter is given by:

$\left|\Psi_{\text {in }}\right\rangle=\mathrm{e}^{\mathrm{i}\left(k_{i} l_{\uparrow}+k_{s} l_{\leftrightarrow}\right)} c\left|\uparrow \omega_{i}, \leftrightarrow \omega_{s}\right\rangle+\mathrm{e}^{\mathrm{i}\left(\phi+k_{i} l_{\leftrightarrow}+k_{s} l_{\uparrow}\right)} d\left|\leftrightarrow \omega_{i}, \uparrow \omega_{s}\right\rangle$

The phases contain the propagation distances $l_{\uparrow}, l_{\leftrightarrow}$ for the s-polarized and p-polarized photons as well as the wave numbers $k_{i}=\frac{\omega_{i}}{c_{0}}, k_{s}=\frac{\omega_{s}}{c_{0}}$. At a lossless $50 / 50$ beam splitter the mode transformation for the different polarization directions is given by [11]

$$
a_{\uparrow}^{\dagger}=\frac{1}{\sqrt{2}}\left(b_{\uparrow}^{\dagger(1)}+b_{\uparrow}^{\dagger(2)}\right) \quad a_{\leftrightarrow}^{\dagger}=\frac{1}{\sqrt{2}}\left(b_{\leftrightarrow}^{\dagger(1)}-b_{\leftrightarrow}^{\dagger(2)}\right),
$$

for either wavelength, with different signs originating from the Fresnel formulae. The output state of the beam splitter at the detectors 1 and 2 can be calculated to be

$$
\begin{aligned}
\left|\Psi_{\text {out }}\right\rangle= & \frac{c}{2} \mathrm{e}^{\mathrm{i}\left(k_{i} l_{\leftrightarrow}+k_{s} l_{\uparrow}\right)}\left(b_{\uparrow, i}^{\dagger(1)}+b_{\uparrow, i}^{\dagger(2)}\right)\left(b_{\leftrightarrow}^{\dagger(1)}-b_{\leftrightarrow}^{\dagger(2)}\right)|0\rangle \\
& +\frac{d}{2} \mathrm{e}^{\mathrm{i}\left(\phi+k_{i} l_{\uparrow}+k_{s} l_{\leftrightarrow}\right)}\left(b_{\leftrightarrow, i}^{\dagger(1)}-b_{\leftrightarrow, i}^{\dagger(2)}\right)\left(b_{\downarrow, s}^{\dagger(1)}+b_{\leftrightarrow, i}^{\dagger(2)}\right)|0\rangle .
\end{aligned}
$$

By applying the transformation of parallel analyzers under $45^{\circ}, b_{\leftrightarrow}^{\dagger}=\left(b_{\nearrow}^{\dagger}+b_{\nwarrow}^{\dagger}\right) / \sqrt{2}, b_{\uparrow}^{\dagger}=\left(b_{\nearrow}^{\dagger}-b_{\nwarrow}^{\dagger}\right) / \sqrt{2}$, and considering only creation operators $b_{\nearrow}^{\dagger}$, i.e parallel analyzer settings, the coincidence count rate yields

$$
\begin{gathered}
N_{c} \propto\left|\mathrm{e}^{\mathrm{i}\left(k_{i} l_{\uparrow}+k_{s} l_{\hookleftarrow}\right)} c-\mathrm{e}^{\mathrm{i}\left(\phi+k_{i} l_{\hookleftarrow}+k_{s} l_{\uparrow}\right)} d\right|^{2} \\
=|c|^{2}+|d|^{2}-2 c d \cos (\Delta k \Delta z-\phi)
\end{gathered}
$$

with $\Delta k=k_{i}-k_{s}$ and $\Delta z=l_{\uparrow}-l_{\leftrightarrow}$ leading to a spatial oscillation period of $\Delta s=1 / \Delta k$. Thus, in case of more than one entangled mode, the SA interferograms can be explained by a superposition of rates as in Eq. (4) resulting in an overall pattern of

$$
N_{c}(\Delta z) \propto \sum_{i}\left|c_{i}\right|^{2}+\left|d_{i}\right|^{2}-2 c_{i} d_{i} \cos \left(\Delta k_{i} \Delta z_{i}-\phi_{i}\right)
$$

Figure 4d shows such a superposition for four modes depicted in Fig. 4c. Clearly, the green line which is the weighted sum of the individual contributions represents the SA interferogram very well. Thus, the structure of our SA interferograms originates from a superposition of energy-time entangled states generated by different pump modes in the SPDC process.

For the HOMI, the derivation is similar: Instead of Eq. (3), the mode transformations of the state from Eq. (2) at the beam splitter are

$a_{\uparrow}^{\dagger}=\left(b^{\dagger(1)}+b^{\dagger(2)}\right) / \sqrt{2}$ and $a_{\leftrightarrow}^{\dagger}=\left(b^{\dagger(1)}-b^{\dagger(2)}\right) / \sqrt{2}$,

as one polarization needs to be rotated by $90^{\circ}$ before impinging onto the beam splitter. This results in the output state

$$
\begin{aligned}
\left|\Psi_{\text {out }}\right\rangle= & \frac{c}{2} \mathrm{e}^{\mathrm{i}\left(k_{i} l_{\uparrow}+k_{s} l_{\leftrightarrow}\right)}\left(b_{i}^{\dagger(1)}+b_{i}^{\dagger(2)}\right)\left(b_{s}^{\dagger(1)}-b_{s}^{\dagger(2)}\right)|0\rangle \\
& +\frac{d}{2} \mathrm{e}^{\mathrm{i}\left(\phi+k_{i} l_{\leftrightarrow}+k_{s} l_{\ddagger}\right)}\left(b_{i}^{\dagger(1)}-b_{i}^{\dagger(2)}\right)\left(b_{s}^{\dagger(1)}+b_{s}^{\dagger(2)}\right)|0\rangle
\end{aligned}
$$

leading to the same coincidence rate as in Eq. (4).

\section{Photon characterization around $1550 \mathrm{~nm}$}

In the $1550 \mathrm{~nm}$ region, photon pairs were generated by pumping the SPDC crystal with frequency-doubled laser light from an erbium-doped fiber amplifier. The amplifier could be seeded either by $\mathrm{cw}$ light from a frequency-stabilized seed laser at $1550.51 \mathrm{~nm}$ or with nearly transform-limited pulses at $1 \mathrm{MHz}$ repetition rate generated by applying an electro-optic amplitude modulation to the cw seed laser. The frequency-doubled pulses were nearly Gaussian with a pulse width of $3.1 \mathrm{~ns}$. In either case, the crystal for photon pair generation was a $24 \mathrm{~mm}$ long fiber-coupled MgO:PPLN (magnesium oxide-doped periodically poled lithium niobate) ridge waveguide crystal designed for type-II SPDC. The orthogonally polarized photons were separated by a polarizing beam splitter (PBS) for the measurements.

For the characterization of the $1550 \mathrm{~nm}$ photons we implemented three experiments: (1) a grating spectrograph with a different detector suitable for this wavelength range (2) a setup for spectral measurements based on time-of-flight analysis in dispersion-engineered optical fibers (3) an HOM interferometer in the $1550 \mathrm{~nm}$ region employing an all-fiber-component setup.

\subsection{Grating spectrograph}

For photon spectra measurements, a commercial grating spectrograph (Andor Shamrock SR-500i CzernyTurner spectrograph with iDus InGaAs 491 Detector) was set up. In order to reduce thermal noise, the detector needs water cooling and should generally be placed in a cool environment. Thus, the whole spectrograph and detector were enclosed in an inhouse-made insu- 


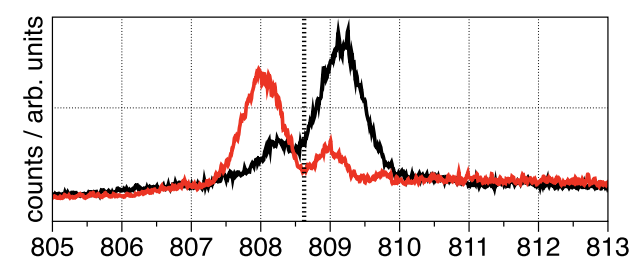

(a)

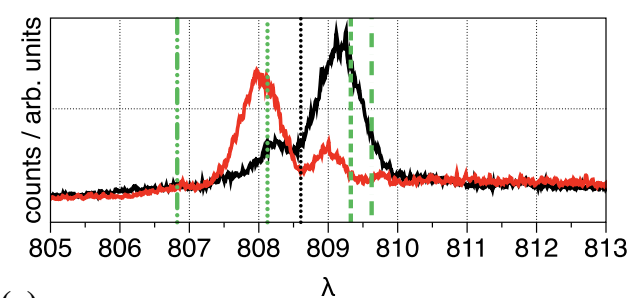

(c)

Fig. 4 a Spectra for the s-polarized and p-polarized SPDC photons. The dashed line indicates the degenerate wavelength for the pump wavelength used b SA interferogram including a fit according to Eq. (5) c Same as in a except

lating box with temperature and humidity control, so that the spectrograph could be cooled down without condensation of water from the air. The box allowed for water cooling of the detector with $1^{\circ} \mathrm{C}$ water temperature and an ambient air temperature of around $8^{\circ} \mathrm{C}$.

With the grating spectograph, the photon spectra of the crystal were measured for different crystal temperatures. As the temperature affects the phase matching of the crystal, the SPDC spectrum shifts with temperature. In Fig. 6a, spectra close to the temperature of $40{ }^{\circ} \mathrm{C}$ for wavelength-degenerate SPDC are shown. For each temperature, signal and idler spectrum were measured separately.

For an ideal crystal, a nearly sinc ${ }^{2}$-shaped spectrum is expected for both signal and idler spectrum [11]. However, the measured spectra show a strong asymmetry with multiple side lobes at one side and only one small side lobe on the other side.

The transverse mode structure of the pump light can influence the photon pair spectra significantly. Nevertheless, the overall spectrum for both signal and idler together is symmetric as expected from energy conservation. Below the main peak for one polarization, a smaller maximum of the other polarization is observed. This is explained by residual photons from the complementary polarization due to non-perfect separation in the fiber-optic polarization beam splitter.

In addition to the grating spectrograph, a setup for spectral measurements via time-of-flight analysis of photons in a medium with high chromatic dispersion was implemented. The chromatic dispersion spectrometer setup uses the signal photons of the produced pairs as a herald and converts the spectral shape of the idler photons into a temporal distribution via propagation in a dispersion-shifted optical fiber of known dispersive power. Similar setups have been demonstrated ear-

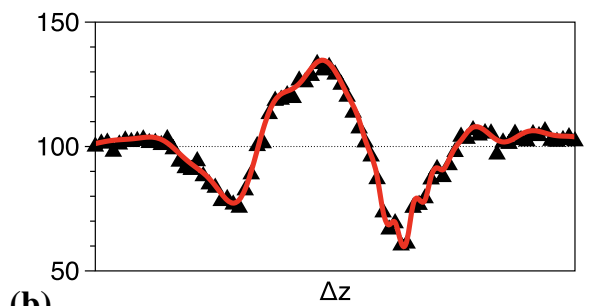

(b)

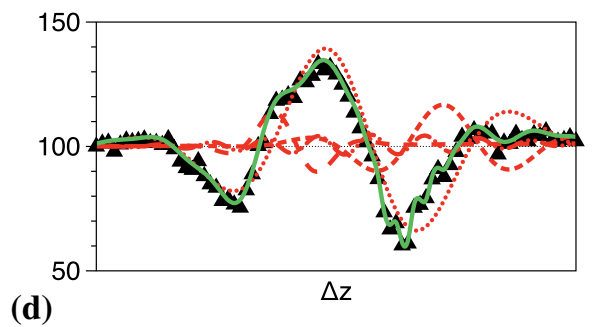

the location of the four modes used in the fit are indicated by vertical lines $\mathbf{d}$ individual contributions of the modes to the SA signal (dashed line) and overall fit as in $\mathbf{b}$ (green line)

Table 1 Parameters for dispersion modules in chromatic dispersion spectrometer setup

\begin{tabular}{lll}
\hline Compensation module for & $30 \mathrm{~km}$ & $60 \mathrm{~km}$ \\
\hline $\begin{array}{l}\text { Dispersion parameter in } \\
\text { ps nm }{ }^{1} \mathrm{~km}^{1}\end{array}$ & $166.6(1)$ & $140.6(1)$ \\
Fiber length in km & $3.157(3)$ & $7.349(1)$ \\
Loss in dB & $2.38(4)$ & $3.5(1)$ \\
\hline
\end{tabular}

lier [12]. Our setup consists of the pulsed photon pair source, a dispersion-engineered optical fiber, two singlephoton detectors and timing electronics. The pulse emission was controlled by the clock of the timing electronics (IDQuantique ID900) that converted the output of the photon detectors (IDQuantique ID220) into timestamps. From the arrival time difference distribution between signal and idler, the photon spectrum was derived from the known dispersion. To further suppress the background, only counts matching the pulse cycle of the SPDC source were counted. Two commercial dispersion compensation modules designed to compensate for chromatic dispersion over $30 \mathrm{~km}$ and $60 \mathrm{~km}$ standard single-mode fiber (SMF-28) were used. Parameters for the modules were measured with commercial OTDR and chromatic dispersion measurement systems (cf. Table 1). First, a single-pass configuration was implemented. The idler photon was sent through the modules and the SPAD was connected directly. Second, a double-pass propagation through the modules was implemented by using a fiber-optic circulator and fiber mirror in order to increase the dispersion (cf. Fig. 5).

The performance of the setup is limited by the photon loss restricting the successful pair detection rate as well as by the timing jitter reducing the temporal and spectral resolution, accordingly. In comparison to the 


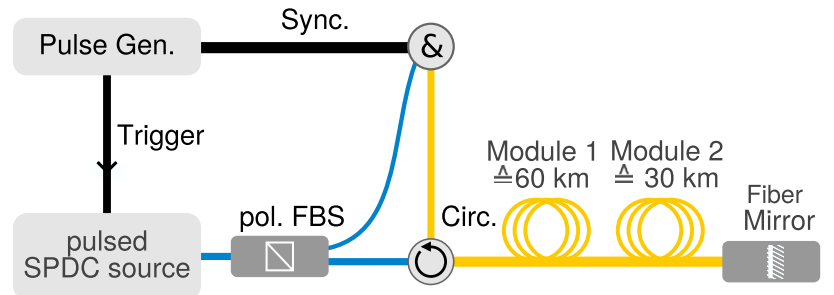

Fig. 5 Chromatic dispersion spectrometer setup (doublepass configuration)

single-pass setup, the double pass increases the spectral resolution at the cost of lower count rates. By using a low mean number of $\mu=0.2$ generated photon pairs per pulse, the multi-pair emission probability was set low enough in order to avoid detection of photons from different pairs in the two detectors. Thus, the temporal resolution is not limited by the pump pulse length of more than $3 \mathrm{~ns}$, but by the jitter of the electronics and photon detectors of around 500 ps.

Figure $6 \mathrm{~b}$ shows a comparison between a spectrum acquired with the spectrograph at $40^{\circ} \mathrm{C}$ crystal temperature and spectra obtained from the chromatic dispersion spectrometer. The acquisition times for both methods differ significantly. Although in principle possible, no deconvolution of the arrival time distribution from the measured distribution and the jitter distribution was applied for resolution enhancement.

\subsection{Hong-Ou-Mandel interference}

For HOM measurements an all-fiber-based setup was implemented (cf. Fig. 7a). First, HOM spectra were measured at the degeneracy temperature of $40.17^{\circ} \mathrm{C}$. The spectra shown in Fig. $7 \mathrm{~b}$ were acquired without any spectral filtering as well as with different band pass

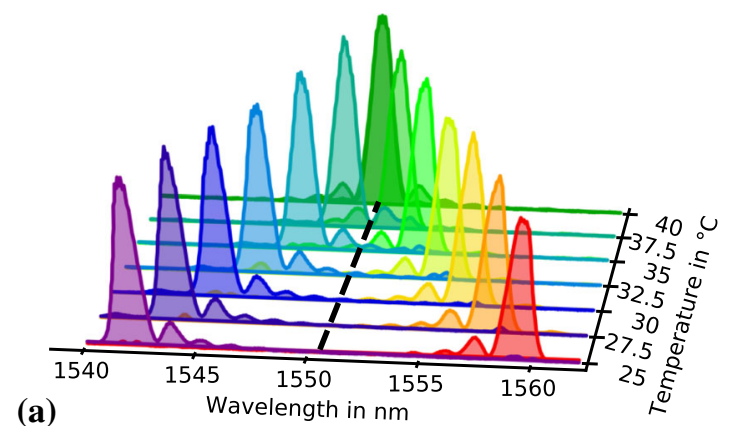

Fig. 6 Spectral measurements in the $1550 \mathrm{~nm}$ region. a Signal and idler spectrum of SPDC from a $24 \mathrm{~mm}$ long fiber-coupled PPLN ridge waveguide crystal pumped with $1.78 \mathrm{~mW} \mathrm{cw}$ laser light at $1550.5 \mathrm{~nm}$. The acquisition time was $400 \mathrm{~s}$ per spectrum. b Comparison between dispersion spectrometer in single-pass and double-pass configuration with a spectrum from the commercial grating spectrograph. The measurement time for the dispersion-based measurements was $12.5 \mathrm{~h}$ at a pulse repetition rate of $20 \mathrm{MHz}$ for filters as noted in the figures. The dip changes with decreasing filter bandwidth from triangular to almost Gaussian as expected. The FWHM of the triangular dip is $0.93 \mathrm{~nm}$. This can be compared with the expected value of

$$
\mathrm{FWHM}_{\text {expd }}=\left.\frac{L c_{0}}{2}\left(\frac{d k_{s}}{d \omega}-\frac{d k_{i}}{d \omega}\right)\right|_{\omega=2 \pi c_{0} / \lambda_{0}}
$$

for an ideal crystal of length $L$ [11]. Plugging in the material-dependent parameters for the crystal used in the experiment, this yields $\mathrm{FWHM}_{\text {expd }}=1.03 \mathrm{~nm}$.

The maximum of the visibility, calculated by dividing minimum and maximum coincidence rate, was $80.6 \%$ for the measurement with the $2 \mathrm{~nm}$ band pass filter. The remaining coincidence counts within the dip can be attributed to different effects. First, the band pass filters were centered at $1550 \mathrm{~nm}$ whereas the central frequency of the photon pairs was at $1550.51 \mathrm{~nm}$. Second, the fiber optical components were connected via mating sleeves, which do not optimally preserve polarization, leading to a few percent of leakage into the fast axis of the PM fibers. Third, the spectra of the photons are intrinsically asymmetric, as can be seen in Fig. 6a. As perfect HOM interference is only expected for indistinguishable photons, all these effects contribute to a decrease in HOM visibility. Narrow-band filtering can be applied in order to make the photon spectra indistinguishable [13]. In order to implement this approach, two fiber Bragg gratings centered at $1550.5 \mathrm{~nm}$ with a FWHM of $0.5 \mathrm{~nm}$ were used. This led to the expected broadening of the dip, but no significant increase in the visibility could be achieved. As the FBG only work for the slow axis of the PM fibers, two different FBGs needed to be inserted into both arms of the interferometer. Taking the different shapes of the FBG transmission curves into account, the maximum achievable

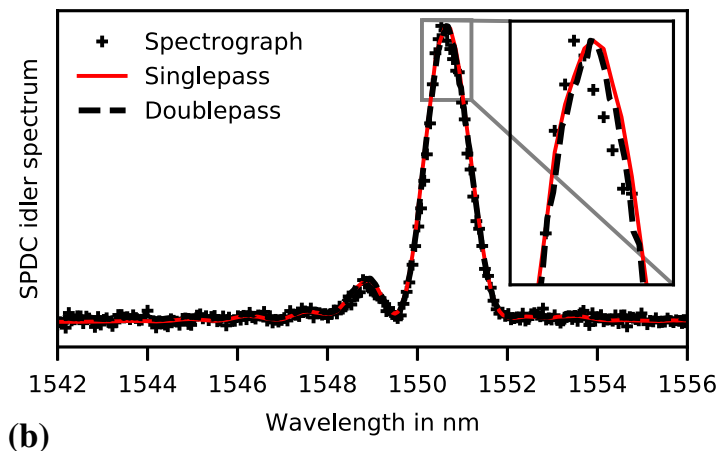

the single-pass and $10 \mathrm{MHz}$ for the double pass with a mean photon pair number of $\mu=0.2$ per pulse. The spectrograph data is the idler spectrum from subfigure (a) at $40{ }^{\circ} \mathrm{C}$ crystal temperature. As the dispersion spectrometer does not provide an absolute value for the wavelength, the maxima were aligned to match. The conversion from arrival time to wavelength for the CDS was performed based on the values from Table 1 


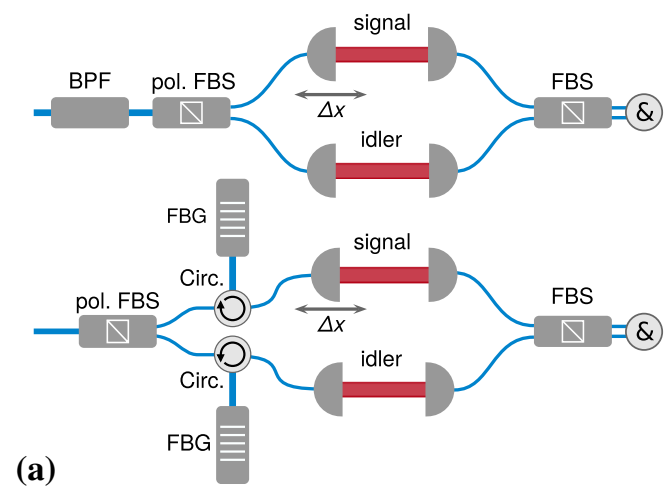

Fig. 7 HOM spectra measurements around $1550 \mathrm{~nm}$. The variable delay line was realized with a piezo linear stage. For the measurements in $\mathbf{b}$ a band pass filter centered at $1550.0 \mathrm{~nm}$, which is slightly different from the central wavelength of $1550.51 \mathrm{~nm}$, was used. Fiber Bragg Gratings (FBG) were used together with circulators for narrow-band

visibility was calculated to $93.3 \%$. The coincidence rate in the plot with FBG filtering in Fig. $7 \mathrm{~b}$ on the lower right decreases at the boundaries as the coupling efficiency of the free-space delay line was optimized only for the central region between $4 \mathrm{~mm}$ and $6 \mathrm{~mm}$.

\section{Summary}

In summary, we have performed experiments on single photons generated by SPDC in waveguides in the wavelength ranges of $808 \mathrm{~nm}$ and $1550 \mathrm{~nm}$ used in our QKD experiments. In both wavelength ranges, we have employed spectrographs capable of single-photon detection sensitivity for a better understanding of the SPDC process. This was crucial to understand the rich structure observed in our $\mathrm{HOM}$ and SA Interferometer experiments for the $808 \mathrm{~nm}$-photons. In the wavelength range around $1550 \mathrm{~nm}$ we performed HOM experiments as well as measurements with a chromatic dispersion spectrometer. We find a significant influence of the spectral properties with the pump mode. As we are employing type-II processes in the SPDC process, a subtle entanglement effect can be observed in the twophoton interference experiments, e.g., in the HOM and SA interferometers as the two-photon interference spectra can be explained by the observation of entangled states due to the photons' spectral properties.

We thank Paul Wagner from Deutsche Telekom Technik $\mathrm{GmbH}$ for loaning the dispersion modules to us. This research was supported by the Deutsche Forschungsgemeinschaft (DFG) as part of the project P4 within CRC 1119 CROSSING.

Funding Information Open Access funding enabled and organized by Projekt DEAL.

Open Access This article is licensed under a Creative Commons Attribution 4.0 International License, which permits use, sharing, adaptation, distribution and reproduction in

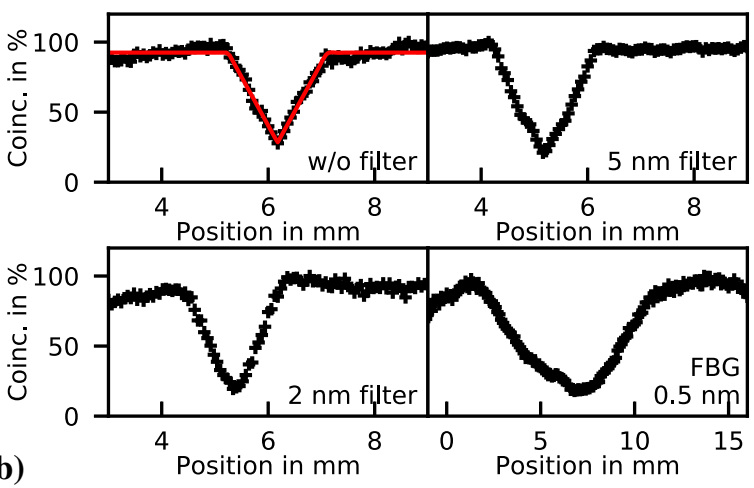

filtering. The difference in the dip positions between the spectra originates from the difference in the optical path length for fast and slow axis of the PM fibers in the band pass filter components. b Hong-Ou-Mandel spectra for photon pairs around $1550.5 \mathrm{~nm}$ with and without filtering at $40.17^{\circ} \mathrm{C}$ crystal temperature

any medium or format, as long as you give appropriate credit to the original author(s) and the source, provide a link to the Creative Commons licence, and indicate if changes were made. The images or other third party material in this article are included in the article's Creative Commons licence, unless indicated otherwise in a credit line to the material. If material is not included in the article's Creative Commons licence and your intended use is not permitted by statutory regulation or exceeds the permitted use, you will need to obtain permission directly from the copyright holder. To view a copy of this licence, visit http://creativecomm ons.org/licenses/by/4.0/.

\section{References}

1. M. Geihs, O. Nikiforov, D. Demirel, A. Sauer, D. Butin, F. Günther, G. Alber, T. Walther, J. Buchmann, The status of quantum-key-distribution-based long-term secure internet communication. IEEE Trans. Sust. Comp. (2019). https://doi.org/10.1109/TSUSC. 2019.2913948

2. ID Quantique SA, 2020. https://www.idquantique.com

3. Toshiba Europe Limited, 2020. https://www. toshiba.eu/eu/Cambridge-Research-Laboratory/ Quantum-Information/Quantum-Key-Distribution

4. I. Marcikic, H. de Riedmatten, W. Tittel, H. Zbinden, M. Legré, N. Gisin, Distribution of time-bin entangled qubits over $50 \mathrm{~km}$ of optical fiber. Phys. Rev. Lett. 93, 180502 (2004)

5. T. Honjo, S.W. Nam, H. Takesue, Q. Zhang, H. Kamada, Y. Nishida, O. Tadanaga, M. Asobe, B. Baek, R. Hadfield, S. Miki, M. Fujiwara, M. Sasaki, Z. Wang, K. Inoue, Y. Yamamoto, Long-distance entanglementbased quantum key distribution over optical fiber. Opt. Exp. 16(23), 19118-19126 (2008)

6. H. Takesue, K.-I. Harada, K. Tamaki, H. Fukuda, T. Tsuchizawa, T. Watanabe, K. Yamada, S.-I. Itabashi, Long-distance entanglement-based quantum key distribution experiment using practical detectors. Opt. Exp. 18(16), 16777-16787 (2010) 
7. X. Liu, X. Yao, H. Wang, H. Li, Z. Wang, L. You, Y. Huang, W. Zhang, Energy-time entanglement-based dispersive optics quantum key distribution over optical fibers of $20 \mathrm{~km}$. Appl. Phys. Lett. 114(14), 141104 (2019)

8. M. Lucamarini et al. Implementation security of quantum cryptography. Technical Report 27, ETSIEuropean Telecommunications Standards Institute, 2018

9. C.K. Hong, Z.Y. Ou, L. Mandel, Measurement of subpicosecond time intervals between two photons by interference. Phys. Rev. Lett. 59, 2044-2046 (1987)
10. Y.H. Shih, C.O. Alley, New type of Einstein-PodolskyRosen-Bohm experiment using pairs of light quanta produced by optical parametric down conversion. Phys. Rev. Lett. 61, 2921-2924 (1988)

11. Z.Y.J. Ou, Multi-Photon Quantum Interference (Springer, US, 2007). 9780387255545

12. K. Zielnicki, K. Garay-Palmett, D. Cruz-Delgado, H. Cruz-Ramirez, M.F. O'Boyle, B. Fang, V.O. Lorenz, A.B. U'Ren, P.G. Kwiat, Joint spectral characterization of photon-pair sources. J. Mod. Opt. 65(10), 1141-1160 (2018)

13. S. Wang, C.-X. Liu, J. Li, Q. Wang, Research on the Hong-Ou-Mandel interference with two independent sources. Sci. Rep. 9(1), 3854 (2019). (ISSN 2045-2322) 